\title{
Impact of COVID-19 on the mental health of surgeons and coping strategies
}

Anusha Balasubramanian, MRCS, MMED (ORL-HNS) ${ }^{1}$

${ }^{1}$ The Royal Marsden NHS Foundation Trust

May 13, 2020

\begin{abstract}
Unprecedented times call for extraordinary measures. While surgeons across the globe try to comprehend the evolving façade of the COVID 19 pandemic and improvise surgical practice to the best of their ability, the psychological impact of the stress on their own mental health and wellbeing has been underestimated. This paper aims to review the indirect and overt factors that may affect the mental health of a surgeon in the present circumstances. Furthermore, it will aim to highlight key coping mechanisms at individual and institutional level, so as to mitigate the negative psychological impact on surgeons.
\end{abstract}

Anusha Balasubramanian, MRCS, MMED (ORL-HNS) ${ }^{1}$

Vigneshwar Paleri, $\mathrm{MSc}^{2}$

Robin Bennett, $\mathrm{PhD}^{2}$

Vinidh Paleri, MS, FRCS (ORL-HNS) ${ }^{1}$

\section{AFFLIATIONS OF AUTHORS :}

1 Head and Neck Unit, The Royal Marsden NHS Foundation Trust, London, UK

2 Lewisham CAMHS, South London and Maudsley NHS Foundation Trust, London, UK

\section{CORRESPONDING AUTHOR :}

Anusha Balasubramanian

Head and Neck Unit,

The Royal Marsden NHS Foundation Trust,

London, UK

Email : htchd766@gmail.com

Tel : +44 7783442155

\section{BRIEF RUNNING TITLE :}

COVID-19 mental health impact on surgeons

\section{KEYWORDS :}

covid-19 impact, mental health, surgeons, otolaryngology, head neck 


\section{CONFLICT OF INTEREST AND FUNDING :}

Nil

\section{DISCLOSURE :}

None

\section{ABSTRACT}

Unprecedented times call for extraordinary measures. While surgeons across the globe try to comprehend the evolving façade of the COVID 19 pandemic and improvise surgical practice to the best of their ability, the psychological impact of the stress on their own mental health and wellbeing has been underestimated. This paper aims to review the indirect and overt factors that may affect the mental health of a surgeon in the present circumstances. Furthermore, it will aim to highlight key coping mechanisms at individual and institutional level, so as to mitigate the negative psychological impact on surgeons.

\section{INTRODUCTION}

Surgical training hones one to be tough, yet calm especially during stressful periods. During these unprecedented times, all clinicians are susceptible to mental stress. Measures should be taken, both at an organisational and at a personal level to provide service while minimising risks to all healthcare professionals.

Irrespective of specialty, healthcare workers fear the reality of the present and uncertainty of the future. It is evident that ORL, oral, maxillo-facial and head and neck surgeons are at significant risk of exposure. While all protective measures must be taken and guidance adhered to consciously at all times, maintaining a well-balanced physical and mental health is of paramount importance to function with optimal clarity.

This paper will focus on consolidating the pre-existing self-care tips and mental health resources, summarise webinars and teleconference proceedings from hard hit areas, and discussions with experts in the field, which will serve as a resource to mitigate the short and long-term psychological effects of the current pandemic.

\section{The Risks of COVID-19 to Surgeons}

As of May $6^{\text {th }}$, 2020, the Johns Hopkins coronavirus resource center had reported 3,689,887 confirmed cases involving 187 countries worldwide, with 258,160 deaths. Data from Italy revealed that up to 20\% of healthcare workers were potentially infected ${ }^{1}$, and as of $29^{\text {th }}$ April 2020, a total of 154 Italian doctors had succumbed. ${ }^{2}$ Surgeons dealing with the upper aerodigestive tract were found to be equally at risk as their counterparts in the emergency room, general ward and intensive care units. ${ }^{3}$ It is notable that the first healthcare workers to succumb of COVID-19 complications in Wuhan, China and UK were both ORL surgeons. ${ }^{4,5}$

High viral loads have been detected in the nose and throat especially after symptom onset. ${ }^{6}$ Thus, both office-based and operating theatre-based procedures such as nasal endoscopy, laryngoscopy, tracheostomy and all transoral interventions are considered to be aerosol generating procedures, and deemed to put the clinician at higher risk of viral infection. In their recent recommendations for the COVID-19 pandemic, the International Head and Neck Scientific Group (IHNSG) have advised to avoid all forms of aerosol generating procedures and that they should be employed only when it is absolutely necessary. ${ }^{3}$

Issues with personal protective equipment (PPE) have been reported worldwide. ${ }^{7}$ While the IHNSG have strongly recommended the use of full PPE for healthcare personnel involved with procedures of the upper aerodigestive tract, ${ }^{3}$ reports of surgical teams having to resort to creating their own PPE using garbage bags, glue, rubber bands and plastic cover of folders bought from stationary shops have emerged. ${ }^{8}$ Health care workers have expressed concerns over acute supply shortages, insufficient time for adequate training and unavailability of mask fitting test. A survey of 258 ENT surgeons was conducted across the UK during the peak of the pandemic in March 2020, seeking their opinion around PPE-related issues. ${ }^{9}$ Among the respondents, $40 \%$ stated that they had not attended a PPE training course or that one was unavailable. More 
worrying was the fact that N95 masks were only available to 27\% of ENT surgeons with $95 \%$ predicting that PPE stocks would deplete in due course. More than half were aware that there was a COVID-19 protocol in place implemented by their Trust. ${ }^{9}$

\section{The Impact of COVID-19 on Mental Health}

Surgeons and trainee doctors from various surgical disciplines have been redeployed to cover on COVID wards and work in the frontline in hotspot areas to help cope with rising demands attributed to the surge in patients. The stress of having to step out of the comfort zone of usual practice into a high-risk unfamiliar environment at a short notice can be overwhelming, as demonstrated by Lai et al where $70 \%$ and $50 \%$ of 1257 healthcare workers (40\% frontline professionals) in China reported symptoms of distress and depression respectively. ${ }^{10}$

Similarly, Liang et al assessed the mental health status of 59 staff associated with COVID departments and other related departments at the Fifth Affiliated Hospital of Sun Yat-sen University using Zung's self-rating depression scale (SDS) and self-rating anxiety scale (SAS). ${ }^{11}$ Although statistically insignificant, younger staff members ([?] 30 years of age) were found to have higher SDS scores. Huang et al conducted a similar assessment of the mental health of frontline clinicians in China. ${ }^{12}$ Across 230 responses, the prevalence of anxiety and stress disorders were $23 \%$ and $27 \%$ respectively; with higher incidence of SAS scores and stress disorders among female clinicians. ${ }^{12}$ On a larger scale, Lv et al found an incidence rate of $34.7 \%$ overall anxiety and $24.8 \%$ of mild anxiety across 8028 surveyed doctors and nurses on the frontline. ${ }^{13}$

Further to this, Kang et al. explored the impact of mental health and coping strategies of Wuhan medical personnel between January 29th and February 4th 2020. ${ }^{14}$ Employing mental health measures for depression (Patient Health Questionnaire-9; PHQ-9), anxiety (Generalised Anxiety Disorder-7; GAD-7) and distress symptoms (Impact of Events Scale-Revised; IES-R) - a cohort of 994 staff responded to the study. Statistically significant results revealed mental health disturbances were prevalent across all participants for the three outcome measures. Individuals reported to be symptomatic across the sub-threshold (36\%), mild $(34.4 \%)$, moderate $(22.4 \%)$ and severe $(6.2 \%)$ levels. Pivotally, coping strategies were varied across all participants ranging from perusing psychological resources $(36.3 \%), 50.4 \%$ accessing digital psychological recommendations (36.3\%) and participating in therapeutic support (17.5\%). Individuals who experienced severe disturbances were less likely to access psychological materials or online mental health guidance. These aforementioned studies not only highlight the necessity for greater and earlier support, but also the versatility of resources that should be provided and promoted to frontline clinicians.

Finally, importance should be given to the social and practical factors associated with living through the social-isolation response to the pandemic. Several facets are adversely impacted inclusive of socialisation with friends and family, regular engagement in leisure activities and procurement of sustenance and other essential items. Fundamentally, there is a detrimental impact on the freedom of movement and a risk of increased anxiety about the health and safety of friends and family. These factors consolidated, may affect the surgeon's ability to manage stress on a daily basis. As normal service resumes in clinics, there is a potential that there could be a surge in referrals of the "worried well" group, leading to increased demand on services and further work stress.

\section{The Role of the Surgeon}

As a team leader, manager or senior clinician, the surgeon is expected to be at his/her best at all times. Personnel who are emotionally distressed may express fear, panic, anger, absenteeism, carelessness in protecting themselves and inadvertently expose others to avoidable risk. In this context, The British Psychological Society's practical guidance outlines the role that is expected of a leader or manager in supporting staffs who are apprehensive or emotionally affected during the COVID-19 pandemic, the various response phases and the principles on responding well to the needs of the staff (Table 1).

In addition to the above, the surgeon is expected to provide holistic person-centred care for outpatients. In the theatre environment, they undertake several responsibilities inclusive of allocating resources optimally, 
mobilising staffs, managing daily issues while attempting to remain calm and composed, push their own thoughts aside, and continually make rational, crucial and critical judgments when required to do so.

This pressure is further mounted by the fear of transmitting infection to family members when they return home. Reports have emerged that many doctors have resorted to isolating themselves from their family so as to protect their loved ones. ${ }^{15}$ The pre-existing emotional stress is further aggravated by self-isolation.

Continual efforts have been made to create pathways, modify working practices especially where resources are severely constrained, as well as create guidance for the clinical management of COVID positive patients and, to adapt the current management protocols in the pandemic setting, as evidenced by the constant updates on the ENT UK website. ${ }^{16}$ However, there still remains the underlying fear, worry, panic, confusion, uncertainty, depression, grief and anxiety in many members of the surgical fraternity which have been overlooked and require equal and immediate attention.

\section{The Dangers of Burnout}

Prior to COVID-19 concerns, reviews have highlighted that up to $54 \%$ of medical professionals in the UK are emotionally exhausted. ${ }^{17}$ Alongside fatigue and frustration, emotional exhaustion is a core symptom of burnout in clinicians. Vijendren et al, found that within a cohort of 108 ENT Surgeons in the UK, $56.5 \%$ of respondents were classified at high risk of developing psychological morbidity, with $28.9 \%$ of reported scores suggesting burnout. ${ }^{18}$ As such, given the additional stresses and disruption caused by COVID-19, clinicians have a greater likelihood of developing burnout in the long-term. ${ }^{19}$

Burnout has several harmful ramifications including substance misuse, absenteeism and medical errors, ${ }^{20-22}$ which can escalate to mental health difficulties such as increased suicidal ideation and depression. ${ }^{23,24}$ Surprisingly, the sole study investigating burnout prevalence in clinicians during COVID-19 epidemic found a lower frequency of burnout in healthcare professionals working on the frontline comparatively to those who were working on wards for uninfected patients. ${ }^{25}$ Several limitations were revealed inclusive of small sample size and selection bias of responses (i.e. distressed medical professionals may have not responded to the survey). Critically, the authors point out that the timing of their study (March 2020) was not a peak period for COVID-19 in Wuhan, China.

Furthermore, a cross-sectional study by Cai et al explored the psychological burden and coping strategies over 500 frontline medical staff in Hunan. Specifically, researchers found the core facets associated with stress were concerns for their families, personal and patient safety. Comparatively to other healthcare workers, doctors felt a higher degree of social and moral responsibility in their vocation and greater scores of anxiety associated with working overtime. ${ }^{26}$

The aforementioned factors (summarised in Table 2), highlights the detrimental impact of burnout on mental health. A surgeon should ideally be in a strong and stable frame of mind to focus on the work at hand, once the various coping mechanisms have been adopted to deal with possible emotional effects of the current pandemic. Irrespective of specialty, Cai et al discovered that knowledge of COVID-19 prevention and transmission, social isolation measures, exhibiting positive self-attitude and seeking family and peer support were all essential facets that significantly reduced stress and burnout in clinicians. ${ }^{26}$ Hereinafter, key coping strategies as recommended by health services are discussed in greater depth.

\section{COPING STRATEGIES}

\section{Positive Lifestyle Behaviours}

Capitalising on coping strategies akin to positive lifestyle behaviours can vastly improve mental health wellbeing. These activities which include eating healthy food, engaging in regular physical activity, practicing good sleep hygiene where possible and guaranteeing sufficient rest between shifts have been recommended by the World Health Organisation (WHO). ${ }^{27}$ Furthermore, avoidance of harmful coping strategies such as alcohol and drug misuse, consistent rumination about COVID-19 or engaging in high-risk behaviours (i.e. 
gambling / excessive spending) is pivotal due to these activities being more damaging in the long-term. Alternatively, social media use for the purpose of maintaining social contact can be considered as a positive lifestyle behaviour. However, consuming excessive media coverage pertinent to COVID-19 may impact negatively on mental health, comparably to the relationship found between viewing disaster media coverage and adverse psychological outcomes. ${ }^{28}$

\section{Mindfulness}

In addition to positive lifestyle changes, compliance with mindfulness-based programmes have been shown to reduce symptoms of burnout in doctors. ${ }^{29}$ Lebares et al's cross-sectional study indicated dispositional mindfulness was associated with a decreased risk of symptoms associated with burnout, distress and severe stress in surgeons. ${ }^{30}$ Similarly, research has shown that specific relaxation techniques (i.e. progressive muscle relaxation) based on elements in mindfulness has been shown to reduce symptoms of anxiety and promote sleep quality in patients with COVID-19. ${ }^{31}$

In this age of exponential technological progress, mobile-health apps are being increasingly used as a source to enhance personal wellbeing. Mindfulness apps have been shown to significantly strengthen resilience and reduce burnout in medical trainees. ${ }^{32}$ The American Psychiatric Association's "app evaluation model" is a framework that rates the efficacy and dangers of mobile and online apps. ${ }^{33}$ This is in line with ORCHA (Organisation for the Review of Care and Health Apps), a health app evaluation and advisor organisation for NHS Digital and NHS England, who have recently launched a COVID-19 specific health-app formulary supporting healthcare professionals and patients. (Table 1) Pospos et al, reviewed data on the effectiveness of these key apps used by clinicians and the general public, recommending the following for specific circumstances: meditation (Headspace, guided meditation audios), suicide prevention (Stay Alive, Virtual Hope box), breathing (Breath2Relax) and Web-based Cognitive Behavioural Therapy (MoodGYM, Stress Gym) $\cdot{ }^{34}$ Specifically, the MoodGYM app has been shown to decrease suicidal ideation amongst medical interns. ${ }^{35}$ Given the ease and accessibility of the aforementioned apps, these mindfulness-based platforms act as a virtual space to disconnect, de-stress and strengthen resilience. ${ }^{36,37}$

\section{EXTERNAL RESOURCES}

The following sections will seek to provide helpful resources for clinicians and highlight novel approaches for institutions to consider during this current climate. It should be noted that whilst extensive, the list of materials is not exhaustive. Consistent modifications to resources are being provided by several websites and services, and the most up-to-date advice should be prioritised.

\section{Supporting the Individual}

The Academy of Medical Royal Colleges have dedicated a page on self-care tips and resources to utilize during the pandemic, some of which have been summarised in Table 3 below. ( Table 1 )

Several validated scales can be employed to assess one's own mental health state. The most prevalent outcome measure in the UK for depression and anxiety would be the PHQ-9 \& GAD-7 respectively. Further notice should be given to IES-R as well; a short assessment for traumatic stress symptoms. Alternative measures also include Zung's self-rating depression scale (SDS) and self-rating anxiety scale (SAS). All questionnaires are highlighted below (Table 1 ).

If clinicians struggle with regular compliance for mindfulness apps - practical steps have been created that can be followed, specifically during this period. FACE COVID is a 9-step programme that healthcare professionals can hold in mind, based on key principles of Acceptance and Commitment Therapy. ${ }^{38}$ Among the steps outlined involve acknowledging one's thoughts, identifying available resources and instituting the appropriate measures. (Table 1 )

\section{Support from the Institution}

Healthcare provider organisations and institutions have a crucial role to play in terms of supporting healthcare staffs of all backgrounds in dealing with the emotional effects of the pandemic. Financial, digital platform, 
logistics and psychological services support may be required according to the needs of every individual. Key mechanisms will be discussed that could be implemented across hospital sites that may prove useful to individuals on managing their mental health wellbeing during the COVID-19 pandemic.

Schwartz rounds are an evidence-based forum that allows a group of staffs of all backgrounds to reflect and discuss their emotions related to work matters. ${ }^{39}$ The positive impact of the Schwartz rounds has been demonstrated, with feedback indicating that emotions were acknowledged, validated and staff members took pride in being a member of a strong supportive team. ${ }^{40.41}$ In light of the present pandemic, this forum can be held on a virtual platform.

Greenberg and colleagues have described measures that may be implemented by healthcare managers and individuals in supervisory roles to promote and protect mental health among staffs. This includes utilisation and enrolling staffs into pre-existing institution-based peer support programmes. ${ }^{41}$

Considerations regarding a shift rota system in place of the regular daily routine may be beneficial for healthcare professionals. ${ }^{42}$ Breaks from the hospital environment for surgeons can be implemented in addition to assembling a secondary workforce from home, should a colleague need to be replaced. Furthermore, psychology consultation and counselling services that are presently being converted into telemedicine services via digital platforms in many institutions should be easily accessible to staffs and surgeons alike. ${ }^{43}$ Reiteration on maintenance of confidentiality should be emphasised as advocated by The Royal College of Psychiatrists. (table 1)

Chen et al found that mental health can be strengthened creatively by setting up specific teams designed to provide psychologic support (i.e. an assistance hotline team, an interventions team for stress-relief activities and a medical team providing online courses). ${ }^{44}$ However, the authors stress on the importance of key resources that should be provided by hospitals (i.e. comfortable place for rest, necessary PPE training and access to COVID-19 guidance and recommendations). These measures were implemented at the Second Xiangya Hospital of Central South University, the largest tertiary hospital in the epicentre of the Hunan province to support their staffs during the peak of the pandemic. ${ }^{44}$ The relevant measures described above have been summarized in Table 4.

\section{CONCLUSION}

Healthcare professionals and provider institutions have a self and corporate responsibility to ensure that the mental health aspect of all staff is adequately monitored and addressed especially during times of global crisis. Long term mental health issues among healthcare professionals as a result of the pandemic should be minimized. The stigma associated with mental health may result in internalising unhelpful thoughts and emotions. Early recognition of symptoms of low mood and anxiety allows for interventions from friends, family and the organisation. This cycle of support can continue to allow for safer and productive outcomes for surgeons in the workplace. It is imperative that the importance of mental health is championed during the COVID-19 pandemic.

\section{REFERENCES}

1. Remuzzi A, Remuzzi G. COVID-19 and Italy: what next?. The Lancet. 2020;395(10231):1225-1228.

2.https://portale.fnomceo.it/elenco-dei-medici-caduti-nel-corso-dellepidemia-di-covid-19/, accessed April 20, 2020 .

3. Kowalski, LP, Sanabria, A, Ridge, JA, et al. COVID-19 pandemic: Effects and evidence-based recommendations for otolaryngology and head and neck surgery practice. Head $\mathscr{E}$ Neck . 2020; 1- 9.

4. Chan JYK, Wong EWY, Lam W. Practical Aspects of Otolaryngologic clinical services during the 2019 novel coronavirus epidemic: an experience in Hong Kong. JAMA Otolaryngol Head Neck Surg. Published online March 20, 2020. doi:10.1001/jamaoto.2020.0488. 
5.https://www.theguardian.com/world/2020/mar/29/worrying-event-deaths-of-nhs-doctors-from-covid-19stark-reminder-of-pandemic,accessed April 7, 2020.

6. Zou L, Ruan F, Huang M, et al. SARS-CoV-2 viral load in upper respiratory specimens of infected patients. N Engl J Med .2020;382:1177-1179.

7.https://www.who.int/news-room/detail/03-03-2020-shortage-of-personal-protective-equipmentendangering-health-workers-worldwide, accessed April 19, 2020.

8. Bowden E, Campanile C, Golding B. Worker at NYC hospital where nurses wear trash bags as protection dies from coronavirus. New York Post. 25 March, 2020.https:/nypost.com/2020/03/25/worker-at-nychospital-where-nurses-wear-trash-bags-as-protection-dies-from-coronavirus/, accessed April 15, 2020.

9. Davis A, O’Donoghue G. Protect our healthcare workers. BMJ . 2020;369:m1324.

10. Lai J, Ma S, Wang Y et al. Factors associated with mental health outcomes among health careworkers exposed to coronavirus disease 2019. JAMA Network Open . 2020;3(3):e203976.

11. Liang Y, Chen M, Zheng X, Liu J. Screening for Chinese medical staff mental health by SDS and SAS during the outbreak of COVID-19. J Psychosom Res . 2020;133:110102.

12. Huang JZ, Han MF, Luo TD, Ren AK, Zhou XP. Mental health survey of 230 medical staff in a tertiary infectious disease hospital for COVID-19. Chinese journal of industrial hygiene and occupational diseases. 2020 Mar 4;38:E001-.

13. Lv Y, Zhang Z, Zeng W, Li J, Wang X, Luo GQ. Anxiety and Depression Survey of Chinese Medical Staff Before and During COVID-19 Defense. Available at SSRN 3551350. 2020 Mar 7.https://ssrn.com/abstract=3551350, accessed April 14, 2020.

14. Kang L, Ma S, Chen M, Yang J, Wang Y, Li R, Yao L, Bai H, Cai Z, Yang B.X. and Hu S, 2020. Impact on mental health and perceptions of psychological care among medical and nursing staff in Wuhan during the 2019 novel coronavirus disease outbreak: A cross-sectional study.Brain, behavior, and immunity . 2020 Mar 30 .

15.https://www.dailymail.co.uk/femail/article-8136037/Doctors-isolating-FAMILIES-prevent-spreadCOVID-19.html, accessed April 19, 2020.

16. ENT UK Covid 19 page.https://www.entuk.org/covid-19, accessed April 21, 2020.

17. Imo UO. Burnout and psychiatric morbidity among doctors in the UK: a systematic literature review of prevalence and associated factors. BJPsych bulletin. 2017 Aug;41(4):197-204. Accessed April 16, 2020.

18. Vijendren A, Yung M, Shiralkar U. Are ENT surgeons in the UK at risk of stress, psychological morbidities and burnout? A national questionnaire survey. The Ssurgeon . 2018 Feb 1;16(1): 12-9.

19. Dewey C, Hingle S, Goelz E, Linzer M. Supporting clinicians during the COVID-19 pandemic [published online ahead of print, 2020 Mar 20]. Ann Intern Med . 2020;M20-1033.

20. Dyrbye LN, Shanafelt TD, Balch CM et al. Relationship between work-home conflicts and burnout among American surgeons: a comparison by sex. Arch Surg . 2011;146(2):211-217.

21. Oreskovich MR, Kaups KL, Balch CM, et al. Prevalence of alcohol use disorders among American surgeons. Arch Surg . 2012;147(2):168-174.

22. Shanafelt TD, Balch CM, Bechamps G, et al. Burnout and medical errors among American surgeons. Ann Surg . 2010;251(6):995-1000.

23. Shanafelt TD, Balch CM, Dyrbye L, et al. Special report: suicidal ideation among American surgeons. Arch Surg . 2011;146(1):54-62. 
24. Dimou FM, Eckelbarger D, Riall TS. Surgeon burnout: A systematic review. J Am Coll Surg . 2016;222(6):1230-1239.

25. Wu Y, Wang J, Luo C, et al. A comparison of burnout frequency among oncology physicians and nurses working on the front lines and usual wards during the COVID-19 epidemic in Wuhan, China [published online ahead of print, 2020 Apr 10]. J Pain Symptom Manage . 2020;S0885-3924(20)30205-0.

26. Cai H, Tu B, Ma J, Chen L, Fu L, Jiang Y, Zhuang Q. Psychological Impact and Coping Strategies of Frontline Medical Staff in Hunan Between January and March 2020 During the Outbreak of Coronavirus Disease 2019 (COVID-19) in Hubei, China. Med Sci Monit . 2020 Apr 15;26.

27. World Health Organization. Mental health and psychosocial considerations during the COVID-19 outbreak, 18 March 2020. World Health Organization; 2020.

28. Pfefferbaum B, Newman E, Nelson SD, Nitiema P, Pfefferbaum RL, Rahman A. Disaster media coverage and psychological outcomes: descriptive findings in the extant research. Current psychiatry reports. 2014 Sep 1;16(9):464.

29. Krasner MS, Epstein RM, Beckman H, Suchman AL, Chapman B, Mooney CJ, Quill TE. Association of an educational program in mindful communication with burnout, empathy, and attitudes among primary care physicians.JAMA . 2009 Sep 23;302(12):1284-1293.

30. Lebares CC, Guvva EV, Ascher NL et al. Burnout and stress among us surgery residents: Psychological distress and resilience. J Am Coll Surg . 2018;226(1):80-90.

\section{Liu K, Chen Y, Wu D, Lin R, Wang Z, Pan L. Effects of progressive muscle relaxation on anxiety and sleep quality in patients with COVID-19. Comple- mentary Therapies in Clinical Practice . 2020 Mar 6:101132.}

32. Yeo CJ, Barbieri A, Roman G et al. Using smartphone mindfulness apps to increase trainee resilience and reduce burnout. Neurology . 2019;92 (15 Supplement) P2.9-005.

33. American Psychiatric Association. App Evaluation Model. 2017. URL: https://www.psychiatry.org/psychiatrists/practice/mental-health-apps/app-evaluation-model. Accessed April 17, 2020

34. Pospos S, Young IT, Downs N, et al. Web-based tools and mobile applications to mitigate burnout, depression, and suicidality among healthcare students and professionals: A systematic review. Acad Psychiatry . 2018;42(1):109-120.

35. Guille C, Zhao Z, Krystal J et al. Web-based cognitive behavioral therapy intervention for the prevention of suicidal ideation in medical interns: A randomized clinical trial. JAMA Psychiatry . 2015;72(12):11921198.

36. Ho CS, Chee CY, Ho RC. mental health strategies to combat the psychological impact of COVID-19 beyond paranoia and panic. Ann Acad Med Singapore . 2020;49(3):155-160.

37. Bansal P, Bingemann TA, Greenhawt M, Mosnaim G, Nanda A, Oppenheimer J, Sharma H, Stukus D, Shaker M. Clinician Wellness During the COVID-19 Pandemic: Extraordinary Times and Unusual Challenges for the Allergist/Immunologist. J Allergy Clin Immunol: In Practice . 2020 Apr 4.

38. FACE COVID' How to respond effectively to the Corona crisis by Dr Russ Harris, author of The Happiness Trap.https://www.baps.org.uk/content/uploads/2020/03/FACE-COVID-by-Russ-Harris-pdfpdf.pdf.Accessed April 16, 2020.

39. Lown BA, Manning CF. The Schwartz Center Rounds: evaluation of an interdisciplinary approach to enhancing patient-centered communication, teamwork, and provider support. Acad Med . 2010;85(6):10731081. 
40. Sanghavi DM. What makes for a compassionate patient-caregiver relationship?. Jt Comm J Qual Patient Saf . 2006;32(5):283-292.

41. Greenberg N, Docherty M, Gnanapragasam S, Wessely S. Managing mental health challenges faced by healthcare workers during covid-19 pandemic. BMJ . 2020;368:m1211.

42.https://www.rcpch.ac.uk/resources/covid-19-guidance-planning-paediatric-staffing-rotas\#rotas, accessed April 20, 2020.

43. Ayanian JZ. Mental health needs of health care workers providing frontline COVID-19 care. JAMA Health Forum 2020.https://jamanetwork.com/channels/health-forum/fullarticle/2764228, accessed April 9,2020.

44. Chen Q, Liang M, Li Y, et al. Mental health care for medical staff in China during the COVID-19 outbreak. Lancet Psychiatry . 2020;7(4):e15-e16.

\section{Hosted file}

HN_tables.docx available at https://authorea.com/users/312328/articles/450604-impact-ofcovid-19-on-the-mental-health-of-surgeons-and-coping-strategies 